\title{
EDITORIAL
}

\section{Elapsed time of light: science \& applications}

\author{
Light: Science \& Applications (2016) 5, e16021; doi:10.1038/lsa.2016.21; published online 1 January 2016
}

I n 2015, Light: Science \& Applications (LSA) has reached a new milestone: receiving the second impact factor of 14.603. LSA is now listed as the second journal among all journals in the field of optics according to the 2014 Journal Citation Reports from Thomson Reuters. Entering into the fifth-year online, LSA has meanwhile received over 500 submitted papers from 40 countries in 2015, with an increasing tendency. LSA has become a truly international journal with over 50 editors from 10 countries, and the paper submissions received originate from almost 50 different countries for the last 4 years.

This outstanding and unbelievable success can be attributed to the strong commitment and contribution of all the authors, editors, reviewers, readers, editorial staff as well as the organizations behind and their people involved from Changchun Institute of Optics, Fine Mechanics and Physics, Chinese Academy of Sciences and Nature Publishing Group. Therefore, it is our wish to express our sincere thanks for the excellent cooperation among all the entities.

The optics community has well-recognized LSA as a new core journal in its field, resulting in the fact that we receive papers from Nobel laureates and other distinguished researchers, covering a wide spread of topics related to Light: Science \& Applications. Through the recently ended International Year of Light and Light-based Technologies, called by the United Nations Educational, Scientific and Cultural Organization (UNESCO), these technologies and especially their awareness in the public have reached another enhanced status. Browsing through the papers that we have yet published, we can see that almost every day new findings in the field of light, optics, and photonics are discovered. To bring these new and fascinating discoveries to a wider audience, is also one of our main missions. Therefore, we are eager to always receive your newest research results to be submitted and published in Light: Science \& Applications.
Publishing high-quality research is a challenge - which needs enthusiasm and passion in addition to the hard work of all people who are involved. Being on board of the editorial team of LSA right from the first moment fulfils us with pride and happiness. We wish the journal a brighter future, in a way that we can maintain this high level of quality publications and this paramount success. We will be working hard to continue delivering the best papers to the optics community with Light: Science \& Applications!

$$
\begin{array}{r}
\text { Jianlin Cao } \\
\text { Editor-in-Chief, Light: Science \& Applications, } \\
\text { Vice Minister of Science and Technology of } \\
\text { the People's Republic of China. } \\
\text { State Key Laboratory of Applied Optics, CIOMP, CAS, } \\
\text { No. 3888, Dongnanhu Road, Changchun, China } \\
\text { Tianhong Cui } \\
\text { Executive Editor-in-Chief, Light: Science \& Applications, } \\
\text { Professor in Microsystems and Nanoengineering, } \\
\text { University of Minnesota, Minneapolis, MN, USA } \\
\text { Stefan Kaierle } \\
\text { Executive Editor-in-Chief, Light: Science \& Applications, } \\
\text { Laser Center Hannover, Germany }
\end{array}
$$

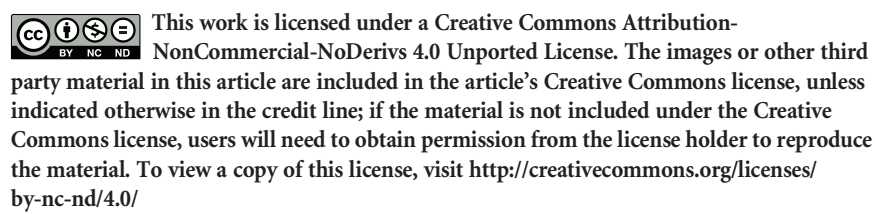

